\title{
Supplement to:
}

\section{Enhanced ice nucleation of simulated sea salt particles with the addition of anthropogenic per- and polyfluoroalkyl substances (PFASs)}

Martin J. Wolf ${ }^{1,2^{*}}$, Yue Zhang ${ }^{3,4,5}$, Jiaqi Zhou ${ }^{4}$, Jason D. Surratt ${ }^{4,6}$, Barbara J. Turpin ${ }^{4}$, and Daniel J. $\mathrm{Cziczo}^{2,7,8}$

1. Yale Center for Environmental Law \& Policy, Yale Law School and Yale School of the Environment, G32 Kroon Hall, 195 Prospect Street, New Haven, CT 06511

2. Department of Earth, Atmospheric, and Planetary Sciences, Massachusetts Institute of Technology, 77 Massachusetts Avenue, Room 54-918, Cambridge, MA 02139

3. Department of Atmospheric Sciences, Texas A\&M University, 3150 TAMU, College Station, Texas 77843

4. Department of Environmental Sciences and Engineering, University of North Carolina at Chapel Hill, 135 Dauer Drive, 166 Rosenau Hall, Chapel Hill, NC 27599

5. Aerodyne Research Incorporated, Center for Aerosol and Cloud Chemistry, 45 Manning Road, Billerica, MA 01821.

6. Department of Chemistry, University of North Carolina at Chapel Hill, Campus Box 3290, Chapel Hill, NC 27599

7. Department of Civil and Environmental Engineering, Massachusetts Institute of Technology, 77 Massachusetts Avenue, Room 1-290, Cambridge, MA 02139Department of Earth, Atmospheric, and Planetary Sciences, Purdue University, 550 Stadium Mall Drive, West Lafayette, IN 47907 

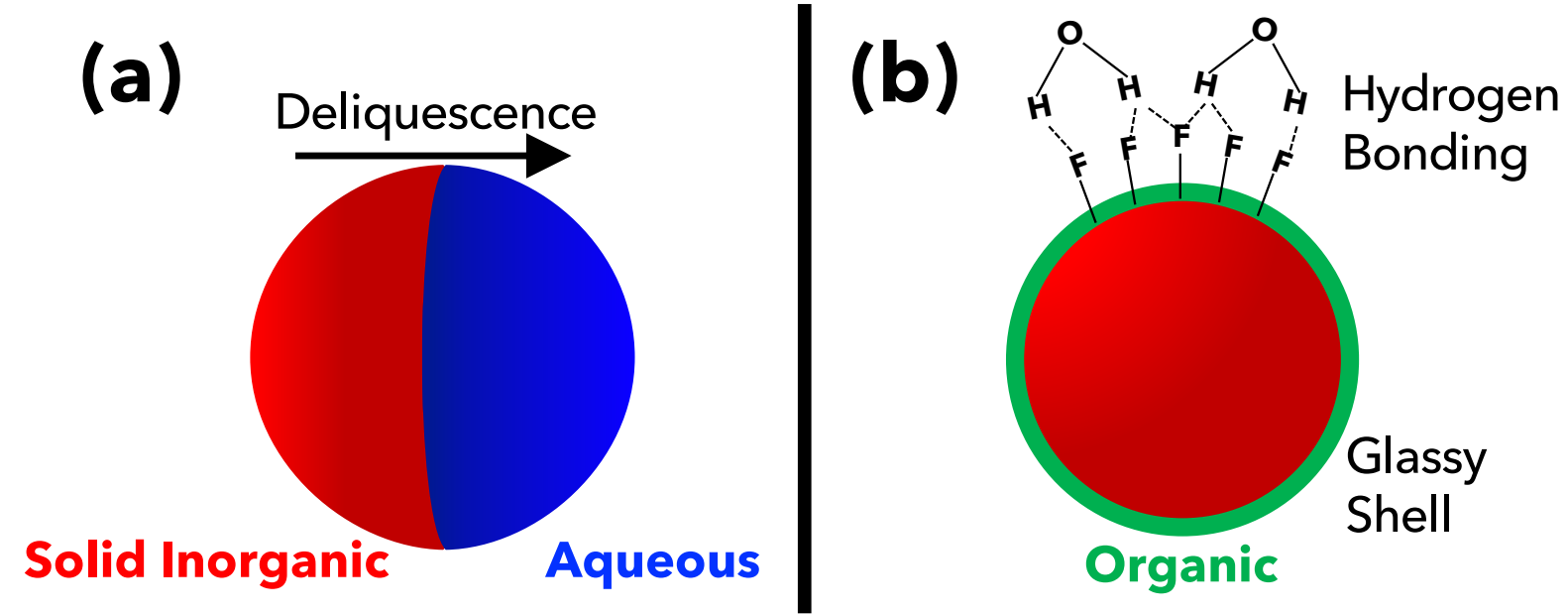

Figure S1. A conceptual framework for the enhanced ice nucleation activity of organic substances. (a) Particles comprised solely of inorganic substances like sea salt can deliquesce, thereby preventing heterogeneously freezing mechanisms like depositional ice nucleation. (b) Internally mixed particles can exhibit core-shell morphologies, where organic coatings can undergo glassy phase transitions at cold temperatures, thereby inhibiting deliquescence of the salty core. Certain organic molecules may also promote ice embryo formation through hydrogen bonding. 


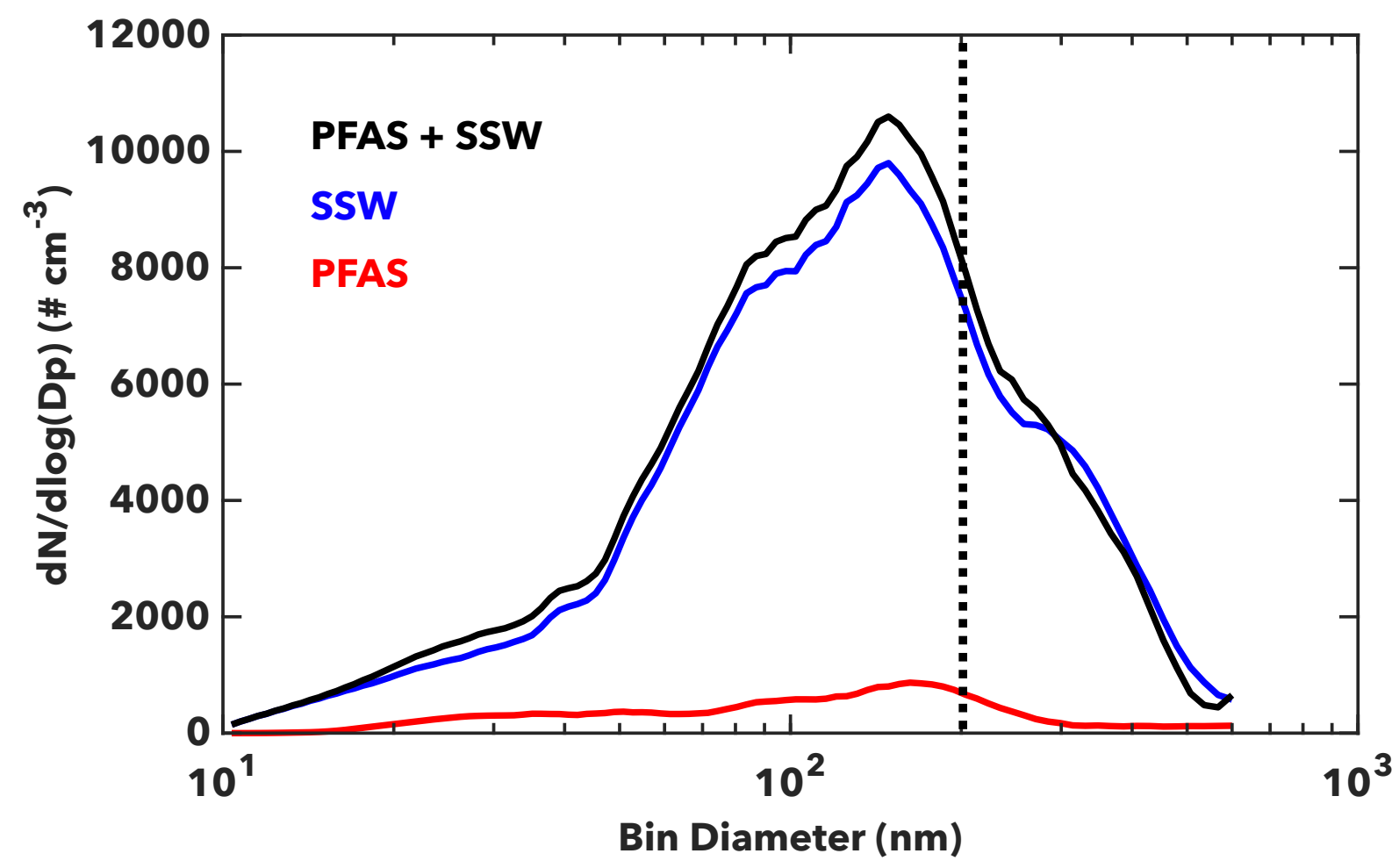

Figure S2. Size distributions of desiccated aerosol particles generated from bubbling PFASs, synthetic seawater (SSW), and PFAS plus SSW solutions. Size distributions represent the average concentrations over 6 scans between 10 and $600 \mathrm{~nm}$. We selected $200 \mathrm{~nm}$ diameter particles - indicated by the black dashed vertical line - on which we performed all ice nucleation experiments. 
Table S1. List of PFAS standards

\begin{tabular}{|c|c|c|}
\hline Analyte & CAS\# & Formula \\
\hline \multicolumn{3}{|l|}{ GenX ("GenX Experiments") } \\
\hline \multicolumn{3}{|l|}{ Mixture of PFASs (“PFASs Experiments”) } \\
\hline \multicolumn{3}{|l|}{ Perfluorocarboxylic acids (PFCAs) - Legacy PFAS } \\
\hline Perfluoro-n-butanoic acid (PFBA) & $375-22-4$ & $\mathrm{C}_{4} \mathrm{HF}_{7} \mathrm{O}_{2}$ \\
\hline Perfluoro-n-pentanoic acid (PFPeA) & $2706-90-3$ & $\mathrm{C}_{5} \mathrm{HF}_{9} \mathrm{O}_{2}$ \\
\hline Perfluoro-n-hexanoic acid (PFHxA) & $307-24-4$ & $\mathrm{C}_{6} \mathrm{HF}_{11} \mathrm{O}_{2}$ \\
\hline Perfluoro-n-heptanoic acid (PFHpA) & $375-85-9$ & $\mathrm{C}_{7} \mathrm{HF}_{13} \mathrm{O}_{2}$ \\
\hline Perfluoro-n-octanoic acid (PFOA) & $335-67-1$ & $\mathrm{C}_{8} \mathrm{HF}_{15} \mathrm{O}_{2}$ \\
\hline Perfluoro-n-nonanoic acid (PFNA) & $375-95-1$ & $\mathrm{C}_{9} \mathrm{HF}_{17} \mathrm{O}_{2}$ \\
\hline Perfluoro-n-decanoic acid (PFDA) & $335-76-2$ & $\mathrm{C}_{10} \mathrm{HF}_{19} \mathrm{O}_{2}$ \\
\hline Perfluoro-n-undecanoic acid (PFUdA) & 2058-94-8 & $\mathrm{C}_{11} \mathrm{HF}_{21} \mathrm{O}_{2}$ \\
\hline Perfluoro-n-dodecanoic acid (PFDoA) & $307-55-1$ & $\mathrm{C}_{12} \mathrm{HF}_{23} \mathrm{O}_{2}$ \\
\hline Perfluoro-n-tridecanoic acid (PFTrDA) & $72629-94-8$ & $\mathrm{C}_{13} \mathrm{HF}_{25} \mathrm{O}_{2}$ \\
\hline Perfluoro-n-tetradecanoic acid (PFTeDA) & $376-06-7$ & $\mathrm{C}_{14} \mathrm{HF}_{27} \mathrm{O}_{2}$ \\
\hline Perfluoro-n-hexadecanoic acid (PFHxDA) & $67905-19-5$ & $\mathrm{C}_{16} \mathrm{HF}_{31} \mathrm{O}_{2}$ \\
\hline Perfluoro-n-octadecanoic acid (PFODA) & $16517-11-6$ & $\mathrm{C}_{18} \mathrm{HF}_{35} \mathrm{O}_{2}$ \\
\hline \multicolumn{3}{|l|}{ Perfluorosulfonic acids (PFSAs) - Legacy PFAS } \\
\hline Perfluorobutane sulfonic acid (PFBS) & $375-73-5$ & $\mathrm{C}_{4} \mathrm{HF}_{9} \mathrm{O}_{3} \mathrm{~S}$ \\
\hline Perfluoropentane sulfonic acid (PFPeS) & 2706-91-4 & $\mathrm{C}_{5} \mathrm{HF}_{11} \mathrm{O}_{3} \mathrm{~S}$ \\
\hline Perfluorohexane sulfonic acid (PFHxS) & $355-46-4$ & $\mathrm{C}_{6} \mathrm{HF}_{13} \mathrm{O}_{3} \mathrm{~S}$ \\
\hline Perfluoroheptane sulfonic acid (PFHpS) & $375-92-8$ & $\mathrm{C}_{7} \mathrm{HF}_{15} \mathrm{O}_{3} \mathrm{~S}$ \\
\hline Perfluorooctane sulfonic acid (PFOS) & $1763-23-1$ & $\mathrm{C}_{8} \mathrm{HF}_{17} \mathrm{O}_{3} \mathrm{~S}$ \\
\hline Perfluorononane sulfonic acid (PFNS) & $68259-12-1$ & $\mathrm{C}_{9} \mathrm{HF}_{19} \mathrm{O}_{3} \mathrm{~S}$ \\
\hline Perfluorodecane sulfonic acid (PFDS) & $335-77-3$ & $\mathrm{C}_{10} \mathrm{HF}_{21} \mathrm{O}_{3} \mathrm{~S}$ \\
\hline Perfluorododecane sulfonic acid (PFDoS) & $79780-39-5$ & $\mathrm{C}_{12} \mathrm{HF}_{25} \mathrm{O}_{3} \mathrm{~S}$ \\
\hline \multicolumn{3}{|c|}{ Perfluoroalkyl ether carboxylic and sulfonic acids (PFECAs and PFESAs) - Emerging PFAS } \\
\hline Perfluoro-2-methoxyacetic acid (PFMOAA) & $674-13-5$ & $\mathrm{C}_{3} \mathrm{HF}_{5} \mathrm{O}_{3}$ \\
\hline Perfluoro-2-methoxypropanoic acid (PMPA) & $13140-29-9$ & $\mathrm{C}_{4} \mathrm{HF}_{7} \mathrm{O}_{3}$ \\
\hline Perfluoro (3,5-dioxahexanoic) acid (PFO2HxA) & $39492-88-1$ & $\mathrm{C}_{4} \mathrm{HF}_{7} \mathrm{O}_{4}$ \\
\hline Perfluoro-2-ethoxypropanoic acid (PEPA) & $267239-61-2$ & $\mathrm{C}_{5} \mathrm{HF}_{9} \mathrm{O}_{3}$ \\
\hline Perfluoro (3,5,7-trioxaoctanoic) acid (PFO3OA) & $39492-89-2$ & $\mathrm{C}_{5} \mathrm{HF}_{9} \mathrm{O}_{5}$ \\
\hline Hexafluoropropylene oxide-dimer acid (HFPO-DA), parent acid of "GenX" & $13252-13-6$ & $\mathrm{C}_{6} \mathrm{HF}_{11} \mathrm{O}_{3}$ \\
\hline Perfluoro (3,5,7,9-tetraoxadecanoic) acid (PFO4DA) & $39492-90-5$ & $\mathrm{C}_{6} \mathrm{HF}_{11} \mathrm{O}_{6}$ \\
\hline Perfluoro 3,5,7,9,11-pentaoxadecanoic acid (PFO5DoA) & $39492-91-6$ & $\mathrm{C}_{7} \mathrm{HF}_{13} \mathrm{O}_{7}$ \\
\hline $\begin{array}{l}\text { 2,2,3,3-tetrafluoro-3-((1,1,1,2,3,3-hexafluoro-3-(1,2,2,2- tetrafluoroethoxy) propan-2- } \\
\text { yl)oxy)propanoic acid (HydroEve) }\end{array}$ & $773804-62-9$ & $\mathrm{C}_{8} \mathrm{H}_{2} \mathrm{~F}_{14} \mathrm{O}_{4}$ \\
\hline 1,1,2,2-tetrafluoro-2-(1,2,2,2-tetrafluoro-ethoxy)ethane sulfonate (NVHOS) & 801209-99-4 & $\mathrm{C}_{4} \mathrm{H}_{2} \mathrm{~F}_{8} \mathrm{O}_{4} \mathrm{~S}$ \\
\hline $\begin{array}{l}\text { 2-[1-[Difluoro[(1,2,2-trifluoroethenyl)oxy]methyl]-1,2,2,2- tetrafluoroethoxy]-1,1,2,2- } \\
\text { tetrafluoroethanesulfonic acid (Nafion Byproduct 1) }\end{array}$ & 29311-67-9 & $\mathrm{C}_{7} \mathrm{HF}_{13} \mathrm{O}_{5} \mathrm{~S}$ \\
\hline $\begin{array}{l}\text { Ethanesulfonic acid, 2-[1-[difluoro(1,2,2,2-tetrafluoroethoxy)methyl]-1,2,2,2- } \\
\text { tetrafluoroethoxy]-1,1,2,2-tetrafluoro- (Nafion Byproduct 2) }\end{array}$ & $749836-20-2$ & $\mathrm{C}_{7} \mathrm{H}_{2} \mathrm{~F}_{14} \mathrm{O}_{5} \mathrm{~S}$ \\
\hline 2,2,3,3,4,5,5,5-4-(1,1,2,2-tetrafluoro-2-sulfoethoxy) pentanoate (Nafion Byproduct 4) & $2416366-18-0$ & $\mathrm{C}_{7} \mathrm{H}_{2} \mathrm{~F}_{12} \mathrm{O}_{6} \mathrm{~S}$ \\
\hline
\end{tabular}

